Observations of asteroids.

The positions of the three new minor planets discovered Febr. 16, 1906, were as follows:

\begin{tabular}{|c|c|c|c|c|c|c|}
\hline Name & 1906 & Gr. m. t. & $\alpha 1906.0$ & 81906.0 & \multicolumn{2}{|c|}{ Daily motion } \\
\hline I906 TJ & Febr. 23 & $14^{\mathrm{h}} 57^{\mathrm{m}} 0^{\mathrm{s}}$ & $9^{h} 55^{m_{2} 856}$ & $+16^{\circ} 32^{\prime} \cdot 5$ & $-63 \cdot 9$ & +0 \\
\hline I $906 \mathrm{TK}$ & $\Rightarrow 23$ & $1457 \quad 0$ & I0 348.2 & +16 45 & -50.0 & \\
\hline $1906 \mathrm{TE}$ & 23 & I 6 I 0 & IO I 743.5 & +1037.6 & -59.6 & +1.7 \\
\hline
\end{tabular}

Taunton, Mass., U.S. A., 1906 Febr. 25.

Foel H. Metcalf.

Beobachtungen von kleinen Planeten und Kometen.

\begin{tabular}{|c|c|c|c|c|c|c|c|c|c|c|c|c|c|}
\hline Objekt & 1906 & & Z. $\mathrm{W}$ & & Gr. & & $\alpha$ al & & $\log p . \Delta$ & & app & & $\log p . \Delta$ \\
\hline (208) Lacrimosa & März 7 & I 2 & $54^{m}$ & $2^{s}$ & 12.0 & I I & $\mathrm{I}^{\mathrm{m}}$ & $25^{5.89}$ & 8.843 & $+6^{\circ}$ & & $24 " 5$ & 0.770 \\
\hline$\gg$ & 8 & I I & 34 & 28 & - & I I & I I & 39.47 & $8.76 g_{n}$ & +6 & 7 & $44 . \mathrm{I}$ & 0.769 \\
\hline (484) Pittsburghia & 7 & 12 & 27 & 45 & I 2.5 & 12 & 36 & $24 \cdot 36$ & 9.078 & +12 & 4 & 35.2 & 0.717 \\
\hline$\gg$ & $\gg \quad 8$ & I 2 & 15 & I & - & I 2 & 35 & 46.35 & $9.125 n$ & +12 & I 3 & $45 \cdot 9$ & 0.717 \\
\hline $1905 \mathrm{SF}$ & Febr. 27 & 8 & 34 & 30 & 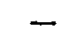 & 4 & 17 & 6.93 & $9 \cdot 44 \mathrm{I}$ & +21 & 18 & 16.3 & $0.65 \mathrm{I}$ \\
\hline 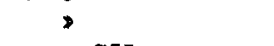 & März 8 & 8 & 33 & 31 & - & 4 & 25 & I $6.2 \mathrm{I}$ & 9.494 & +22 & I & $4 \cdot 4$ & 0.660 \\
\hline $1906 \mathrm{SY}$ & Febr. 25 & I I & 34 & 16 & I 2.0 & 7 & 30 & 41.97 & $9 \cdot 374$ & +15 & 2 & I 6.6 & 0.706 \\
\hline$\gg$ & März 8 & 9 & 43 & 9 & - & 7 & 28 & 49.23 & 9.126 & +14 & $5^{8}$ & $24 \cdot 5$ & 0.688 \\
\hline I $906 \mathrm{TF}$ & Febr. 23 & I 2 & 40 & 47 & I 3.0 & 9 & I I & 47.71 & 9.237 & $+\times 5$ & I 6 & 2.9 & 0.690 \\
\hline$》$ & $\$ \quad 27$ & I 3 & 47 & 20 & - & 9 & 6 & 6.46 & 9.473 & +14 & 20 & 19.8 & 0.728 \\
\hline I906 TG & März 5 & 10 & 33 & 46 & - & 9 & I 5 & 42.52 & 8.196 & $+I_{4}$ & 17 & I 8.8 & 0.689 \\
\hline Komet $1906 \mathrm{~b}$ & , & 9 & 44 & 42 & - & I I & 35 & I 1.56 & $9.443 n$ & $+r$ & 42 & $3 \cdot I$ & 0.807 \\
\hline$»$ & 7 & I 3 & I 9 & I 9 & - & I I & 34 & 16.27 & 8.876 & +1 & 45 & 6.9 & $0.8 \circ 3$ \\
\hline
\end{tabular}

Wien, k. k. Sternwarte, 1906 März 9.

F. Palisa.

\title{
Nachträgliche Entdeckung eines neuen Planeten 1902 HVa*).
}

Beim Nachsuchen älterer Platten habe ich folgende, seiner Zeit übersehenen Planetenspuren gefunden:

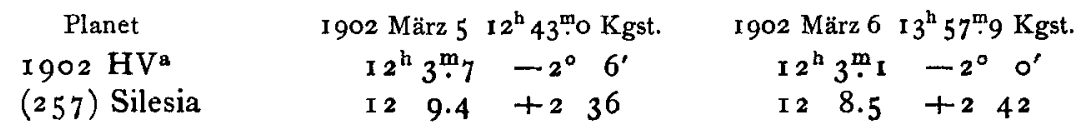

Astrophys. Institut Königstuhl-Heidelberg, r qo6 März 5.

M. Wolf.

*) Da der Planet bei rechtzeitiger Entdeckung zwischen I902 HU und dem später gestrichenen Planeten I902 HV einrangiert worden wäre, ist ihm die Bezeichnung $1902 \mathrm{HVa}$ zuerteilt worden. $K r$.

Photographische Aufnahmen von kleinen Planeten 1906 März 4.

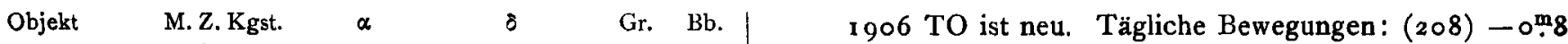
(208) Lacrimosa $15^{\mathrm{h}} 34^{\mathrm{m}} \cdot 5 \times 1^{\mathrm{h}} 14^{\mathrm{m}} \cdot 7+5^{\circ} 50^{\prime}$ I $2.1 \mathrm{~K}+3^{\prime}$, TO - $0^{\mathrm{m}} \cdot 7+9^{\prime},\left(4^{8} 4\right)-0^{\mathrm{m}} \cdot 6+12^{\prime}$. Der Oppositions1906 TO I 555.0 I $228.3+1334$ I3.2 W ort von (208) Lacrimosa in B. J. I 908 ist fehlerhaft. (484) Pittsburghia $》 \quad$ I2 $38 . \mathrm{I}+\mathrm{I}$ I 35 I2.6,$\quad \mathrm{W}=M$. Wolf, $\mathrm{K}=A$. Kopff.

Astrophys. Institut Königstuhl-Heidelberg, 1906 März 5 .

M. Wolf.

\section{Nova 104.1905 Aquilae.}

Professor Frost has furnished the facts which seem to $\mid$ on Sept. 2 I, Sept. 27 , and Oct. 23, 1905, show no nebulosity, prove that the last explanation, given in A. N. 4065 , of the nebulous appearance surrounding Nova Aquilae, No. 2, is the correct one. This appearance is probably due to chromatic aberration. I) Plates taken with the Yerkes 24 -inch reflector 2) Wolf has found a similar effect with other stars having peculiar spectra. 3) If due to nebulosity, its intrinsic brightness must be at least $r 800$ times as bright in proportion to the light of the star, as that around Nova Persei, No. 2.

Harvard College Observatory, I 906 Febr. 3.

Edward C. Pickering. 